\title{
DIFFUSION MODELS FOR MAGNETIC AP STARS
}

\author{
J. BABEL
}

Institut d'Astronomie de l'Université de Lausanne, CH-1290 Chavannesdes-Bois, Switzerland

ABSTRACT Progress made in spectroscopy and in the diffusion theory permits now to make severe comparisons, based on line profiles, between theory and observation.

We first review transport processes which are present in the atmospheric layers of $\mathrm{Ap}$ stars and discuss their relative importance. We then show that mass loss could play a key role for the creation of abundance maps. A mass loss model is proposed for $53 \mathrm{Cam}$ and is compared, by spectrum synthesis, with visible and IUE high resolutions observations. The model accounts well for the line profiles of several elements with an exception for Ti. Furthermore, the abundance stratification predicted by this model gives close agreement with the large variation of the abundances of $\mathrm{Cr}$ and $\mathrm{Fe}$ found between the visible and UV domains. The diffusion-mass loss model finally permits to give a simple interpretation of the peculiar Ca II $\mathrm{K}$ lines observed in many Ap $\mathrm{SrCrEn}$ stars and in particular in $53 \mathrm{Cam}, \beta \mathrm{CrB}$ and $\mathrm{HD} 191742$.

\section{INTRODUCTION}

Recent high signal to noise observations of Ap stars and high quality abundance maps (see Hatzes, Wehlau and Khoklova this meeting) give now the possibility to severely constrain both the diffusion model and the hydrodynamics of the outer layers of magnetic Ap stars. This constitutes a rather unique opportunity to investigate processes which reveal in Ap stars but are certainly present in large parts of the HR diagram (Landstreet 1992).

In this paper, a special emphasis is given to detailed comparisons between results from the diffusion model and observations. Here, we mainly focus on cold Ap stars, as these objects are the most extreme, from the diffusion point of view, and thus the more suitable for a comparison with spectroscopic observations. Our present goal is to determine why or when, together with diffusion, macroscopic hydrodynamic processes are required to explain the observations. We also want to show which observations could be explained from first principles only. 


\section{TRANSPORT PROCESSES}

\section{Diffusion across magnetic field lines}

The most evident effect of the magnetic field on the diffusion velocity of ionised species is the reduction, through Lorentz forces, of the component of the velocity perpendicular to magnetic field lines. For current magnetic field intensities of Ap stars, diffusion velocities can be reduced by more than $50 \%$ only in upper atmospheric layers $\left(\log \tau_{5000}<0.1\right)$ (Michaud et al 1981). The velocity of each ionisation state is reduced in a different way, so that the contribution of each ionisation state to the total diffusion velocity of the species can be very different from the nonnagnetic case. For example, it always enhances the contribution of the neutral state and reduces the contribution of the first ion (state which is the more affected by the magnetic field). Depending on the ionisation states which are dominant in the layer, a horizontal field could lead to a decrease (X II and $\mathrm{X}$ III the dominant ions and $\left.g_{\text {rad }}(X I I)>g_{\text {rad }}(X I I I)\right)$ or increase $\left(\mathrm{XI}_{\mathrm{I}}, \mathrm{X}_{\mathrm{II}}\right.$ and $\left.g_{\mathrm{rad}}(X I)>g_{\mathrm{rad}}(X I I)\right)$ of the effective radiative acceleration on the trace species (Vauclair et al. 1979).

Another consequence of the redistribution of momentum between ionisation states is the appearance of horizontal drifts both along and across magnetic field lines. Such effect was proposed by Michaud et al. (1981) and discussed in details by Mégessier (1984) for Si. She showed that Si could migrate in $\sim 10^{7}$ yrs over the stellar surface. The same effect could also apply to species not supported below the atmosphere (for example: $\mathrm{Ca}, \mathrm{Si}, \mathrm{Sr}, \mathrm{La}$ and $\mathrm{O}$ ). For the elements supported deep in the envelope (like $\mathrm{Cr}$ or Mn, see Babel \& Michaud 1991a (BM91a)), however, it could be shown from simple geometric considerations that the vertical flux always dominates (by factors of $10^{5}$ ) the horizontal flux, so that horizontal drifts can not play any role.

Radiative acceleration in polarised radiative transfer

For the whole range of magnetic fields found in Ap stars (see Landstreet 1992), the effect of the magnetic field on the radiative transfer has to be taken into account as it affects radiative accelerations in the photosphere. The effects of the magnetic field on the radiative transfer are the followings:

- The spectral lines can be desaturated by Zeeman splitting

- The azimuthal, or $\phi$, symmetry is destroyed by a non-vertical magnetic field.

Computations of radiative accelerations thus require to solve the transfer equations for all the Stokes parameters. In LTE and in the absence of anomalous dispersion, the energy absorbed by the lines of an element within mass $d m$, within the angle $d \Omega$, during $d t$ and in the frequency $d \nu$ is (Unno 1956, Eq. 24)

$$
d E=-\kappa_{c}\left(\eta_{I} I_{I}+\eta_{Q} I_{Q}+\eta_{U} I_{U}+\eta_{V} I_{V}\right) d m d \Omega d \nu d t .
$$

The radiative acceleration can then be defined as $g_{\text {rad }}$

$$
g_{\text {rad }}=\frac{2 \kappa_{c}}{c \chi(A)} \int d \Omega n \int_{\nu}\left(1_{o} \Phi_{A}(+n) H\right) d \nu
$$

where the integral is over the outward direction, so that $n$ is only pointing outwards. $\Phi$ is the absorption matrix, $\boldsymbol{H}$ is a generalised Feautrier vector, 
$\chi(A)$ is the mass fraction of element $A$ and $\kappa_{c}$ the continuous opacity (BM91b). The main effects of the magnetic field on the radiative acceleration can be summarised as follows:

- The desaturation of the spectral lines enhances the vertical radiative acceleration with respect to the nonmagnetic case. On separate lines, this amplification can be as large as a factor of 2.5. When one considers all atonic lines of an element $A$, the effect is smaller since only a part of the atomic lines of $A$ are generally desaturated by Zeeman effects. The equilibrium abundance distributions can be enhanced by a factor of $\sim 2$ above $\log \tau_{5000}=-1.0$. Deeper in the atmosphere, Stark broadening progressively limits the relative importance of the Zeeman desaturation, so that for $\log N_{e} \gtrsim 15$ (i.e. below $\log \tau_{5000}=1.0$ in $53 \mathrm{Cam}$ ) magnetic desaturation is ineffective even for magnetic field as large as $10 \mathrm{kG}$.

- A more subtle effect of the magnetic field is the appearance of a horizontal component of the radiative acceleration, when the magnetic field is not vertical. This component, which lies in the plane defined by the vertical and the magnetic field lines, is always directed away from regions where the field is horizontal (BM91b). While this component does not exceed generally $4 \%$ of the vertical component, it can overcome the redistribution effect even for field intensities as low as $500 \mathrm{G}$ (Michaud \& Babel 1991). For Sr (see Figs. 7 and 8 of BM91b), it lies in the opposite direction of the redistribution effects. It drives $\mathrm{Sr}$ particles towards the magnetic poles and could lead to relative overabundances in the polar regions. As noted above, these drifts can however only be efficient for elements not supported below the atmosphere.

Ambipolar diffusion of hydrogen

An important aspect of $\bar{A}$ stars is that hydrogen is partially ionised in their atmosphere. Diffusion of trace elements takes place in a ternary mixture whose major components are protons, electrons and neutral hydrogen. In atmospheric layers, partial pressure gradients of $\mathrm{H}$ and $\mathrm{p}$ differ from their hydrostatic equilibrium value at constant temperature because of temperature gradients (see Michaud \& Babel 1991) As a result, ambipolar diffusion of hydrogen takes place and elertrons and protons move together with respect to hydrogen atoms (Burgers 1969, p.186). The proton velocity $v_{1}$ is given by (BM91c)

$$
\begin{aligned}
& v_{1}=\frac{k T}{m}\left(\frac{n_{0}}{n_{0}+n_{1}}\right)\left(\frac{n_{1}}{n_{1} V_{10}+n_{0} \gamma_{01}}\right) \\
& {\left[-2 \frac{d l n p_{1}}{d r}+\frac{d l n P}{d r}\left(\frac{2 n_{1}+n_{0}}{n_{1}+n_{0}}\right)+\alpha_{1} \frac{d l n T}{d r}\right]}
\end{aligned}
$$

In LTE model atmospheres the proton velocity is directed upwards as $d T / d r<0$. While these velocity drifts are too small to perturb the ionisation equilibrium in the photosphere, they strongly affect the diffusion of ionised trace species. In effect, $v_{1}$ can be much larger than the diffusion velocity of an ionised trace species. Furthermore, ionised trace species exchange much more momentum with protons than with neutral hydrogen, so that the trace species velocity can be approximated by

$$
v_{t} \simeq v_{1}+\hat{v}_{t}
$$




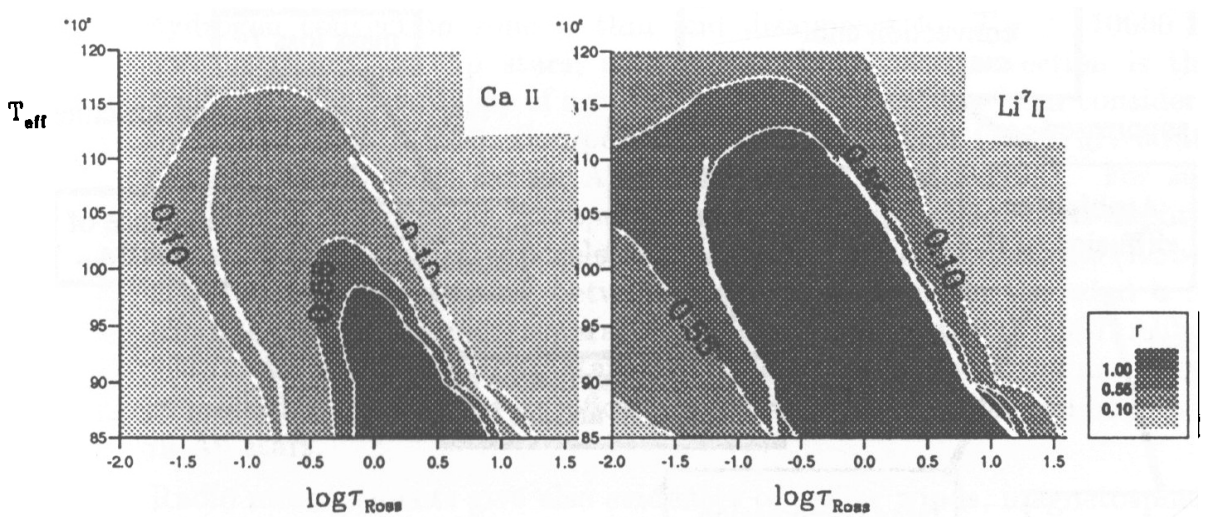

FIGURE I Relative variation, caused by the ambipolar diffusion of hydrogen, of the drift velocity of a $\mathrm{Ca}$ II (left) and of a $\mathrm{Li}^{7}$ II (right). For both species, it has been assumed that $g_{\text {rad }}=0$. The borders of $\mathrm{H}$ II convection, determined from the Schwarzschild criterion, are showned by heavy white lines. The darker aeras are for $r>1$.

where $\hat{v}_{t}$ is the trace species velocity in absence of ambipolar diffusion of hydrogen (the complete expression is given in Eqs. 12 and 33 of BM91c). The left panel of Fig. 1 gives the relative modification $r=\left(\hat{v}_{t}-v_{t}\right) / \hat{v}_{t}$ of the velocity of a $\mathrm{Ca}$ II particle (where $g_{\text {rad }}(\mathrm{Ca} I I)=0$ has been set to zero) as a function of effective temperature and optical depth. We obtain that for stars with $T_{\text {eff }} \lesssim 10000 \mathrm{~K}$, there are layers where $r$ is larger than 1 . This means that in these layers, the momentum transfer through collisions with protons is so large that a $\mathrm{Ca}$ II particle does not need to have any radiative acceleration to be maintained. We also observe in Fig. 1, that the drag efficiency decreases with effective temperature and that the zone of maximum efficiency moves towards smaller optical depths. As the effect on the trace species velocity scales roughly as $Z^{2} / A$, the drag by protons affects much more strongly light ionised particles like $\mathrm{Li}$. In the right panel of Fig. 1 , we observe that $\mathrm{Li}$ could be supported by ambipolar diffusion of hydrogen in a large part of the photosphere. Another interesting aspect is that even outside the domain where convection is expected to occur (from the Schwarzschild criterion), the $\mathrm{Li}$ velocity is strongly affected by ambipolar diffusion. Ambipolar diffusion could thus make the abundance of $\mathrm{Li}^{7}$ (or $\mathrm{Li}^{6}$ ) be much larger than the equilibrium abundance given by $g_{\text {rad }} \simeq g$. It could also enhance the isotopic ratio $\mathrm{Li}^{6} / \mathrm{Li}^{7}$.

The presence of nonvertical magnetic field completely modify the importance of ambipolar diffusion. For large horizontal fields, the drag of trace species is no more affected by the ambipolar diffusion of hydrogen (BM91c). The interaction of ambipolar diffusion with a horizontal magnetic field could, however, directly affect the atmospheric structure and strongly compress the atmosphere in the ionisation region of H (BM91c; Leblanc, Michaud \& Babel in preparation). The effect present similarities with the one described by Peterson \& Theys (1981). 


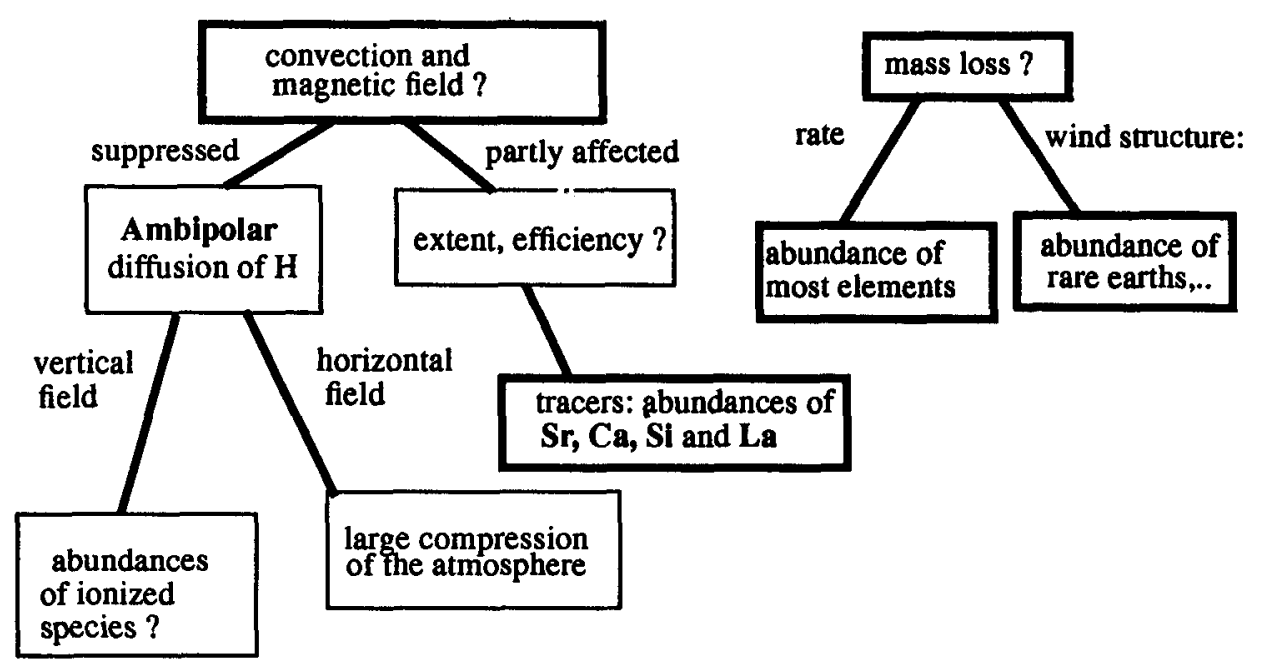

FIGURE II Links between macroscopic processes and photospheric abundances

For nonmagnetic stars, the ambipolar diffusion of hydrogen is not expected to play an important role, as the HII convection, even without overshooting (see Fig. 1 and Holweger in this volume), covers the zone of effectiveness of ambipolar diffusion.

\section{LINKS WITH MACROSCOPIC PROCESSES}

BM91a compared the abundance maps which could be produced by a parameter-free diffusion model to the abundance maps of 53 Cam obtained by Landstreet (1988). They obtained that while they could explain the "mean" observed abundances, the model fails to reproduce the surface abundance inhomogeneities. The impossibility of explaining important features of abundance maps on $53 \mathrm{Cam}$ with the parameter-free diffusion model shows clearly that other hydrodynamical process(es) must be present and affect photospheric abundances. Two processes, poorly known in cold Ap stars, could play a major role:

- Convection and magnetic field: The interaction of convection with magnetic field is not well known. Is it suppressed by the magnetic field, replaced by overstable convection...? The answers are not yet clear. Several attempts are currently done to modelise, with 2-D simulation, the convection of A stars (see Holweger this meeting) or the interaction between convection and external magnetic fields (Fox et al. 1992).

- Mass loss is very common in the HR diagram. For A stars, however, the situation is very puzzling and only upper limits on the mass loss rate have been determined from observations (see Lanz this meeting). The 
driving of mass loss is also not known for A stars. On these stars, the hydrogen convection zone is thin and disappears for $T_{\text {eff }}>10000 \mathrm{~K}$. The existence, on Ap stars, of a corona linked to convection is thus doubtful. The possibility of a radiatively driven wind has been considered by Linsky et al. (1992). Computation based on Abbott (1982) gives rates of $10^{-12.5} \mathrm{M}_{\odot} \mathrm{yr}^{-1}$ for an A2 dwarf (Linsky et al. 1992). For such small rates, however, an extrapolation from the wind of hot stars might be incorrect as the radiative acceleration comes from heavy elements (Abbott 1982 ) and as the coupling between chemical elements in the wind is too small to prevent chemical separation to occur (Michaud et al. 1987, Abbott 1979). More detailed computations including all these effects are clearly requested to tell whether significant radiatively driven winds can be present in Ap stars.

Radio measurements give also evidences of stellar winds, magnetospheres and of nonthermal emission in CP stars hotter than A0. (Brown et al. 1985, Shore et al. 1990, Linsky, Hunger this meeting). No detection have yet been found for classical Ap SrCrEu stars.

In this context, abundance maps could be a very powerful tool to constrain the wind geometry of Ap stars, as surface abundances are very sensitive to the presence of a wind even for rates as low as $10^{-15} \mathrm{M}_{\odot} \mathrm{yr}^{-1}$ (see Vauclair 1975, Michaud et al. 1983, 1987, BM91a and Vauclair et al. 1991)

Fig. 2 shows the interconnection between photospheric abundances and macroscopic processes like convection or mass loss. It also shows which element abundance could be used to trace hydrodynamics. An illustration of the extreme sensitivity of the Sr equilibrium abundance on the locus of the bottom of the $\mathrm{H}$ II convection zone is shown in Fig. 3 for the case of $53 \mathrm{Cam}$. We obtain that a displacement of $1000 \mathrm{~K}$ of the position of the bottom of the convection zone makes the $\mathrm{Sr}$ abundance in the c.z. change by a factor larger than $2 \mathrm{dex}$. The effect is somewhat less important for the apparent $\mathrm{Sr}$ abundance, as the $\mathrm{Sr}$ abundance higher that $\log \tau_{5000}-1.0$ is insensitive to the abundance in the c.z (see below).

\section{A DIFFUSION-MASS LOSS MODEL FOR 53 CAM}

In Babel (1992), we compute the effect of a small inhomogeneous wind on the photospheric abundance of several elements on $53 \mathrm{Cam}$. Chemical elements can be separated in two categories.

- Elements with $g_{r a d}<g$. As the diffusion velocity in the upper atmosphere, due to the hydrogen neutrality, is larger than the mass loss velocity as long as $\dot{M} \lesssim 10^{-12} \mathrm{M}_{\odot} \mathrm{yr}^{-1}$, elements which have $g_{r a d}<g$ in the upper atmosphere cannot be dragged efficiently by the stellar wind. If $g_{\text {rad }}>g$ or $V_{t o t}>0$ in the envelope, they accumulate in the atmosphere reaching there rapidly (about $10^{6} \mathrm{yrs}$ ) an equilibrium solution with $V_{\text {tot }}=0$. The equilibrium condition can be written:

$$
\frac{\partial c_{t}}{\partial r}=c_{t}\left[\frac{1}{D_{t}}\left(V_{w}\left(\Omega_{o}\right)+V_{A}\right)+\frac{A_{t} m_{p}}{k T}\left(g_{r a d, t}\left(c_{t}\right)-g\right)+\ldots\right]
$$




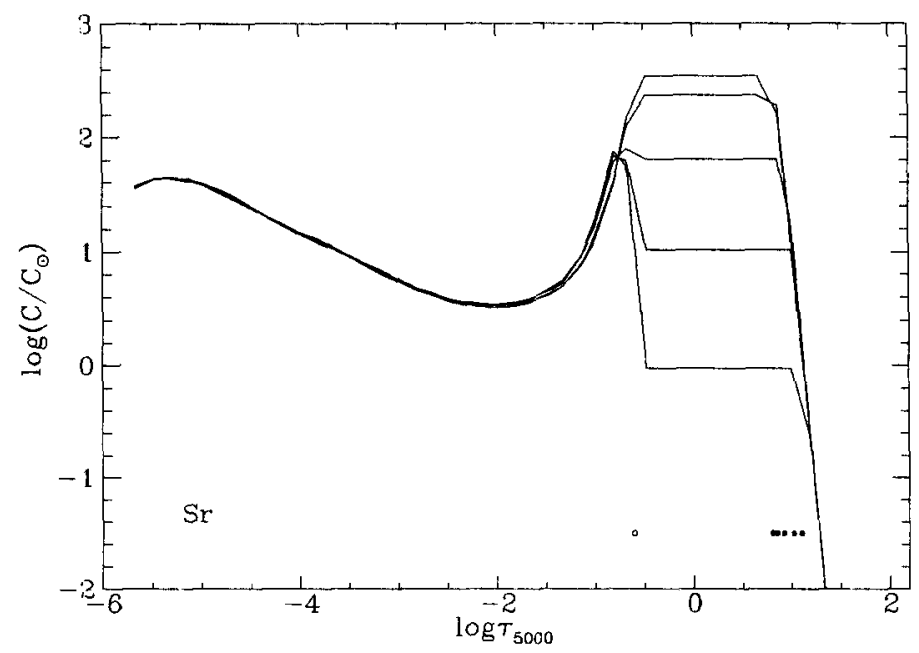

FIGURE III Strontium equilibrium abundance as a function of the location of the bottom of $\mathrm{H}$ II convection. The positions of the bottom of the c.z. are shown by filled circles. The top of c.z is shown by an empty circle. The Sr abundance increases with decreasing convection.

- Elements with $g_{r a d}>g$. In contrast, the outwards velocity of elements which have $g_{r a d}>g$ in the whole atmosphere increases considerably with radius. These elements, like $\mathrm{Ti}$ and rare earths, could reach a stationary solution, characterised by a non-null and quasi-constant outwards flux. The situation for these elements is quite uncertain at present (see below).

\section{Abundance distributions in presence of mass loss}

Equilibrium and stationary abundance distributions were calculated for the atmosphere of $53 \mathrm{Cam}$ and for $\mathrm{Cr}, \mathrm{Sr}, \mathrm{Ca}, \mathrm{Mn}$ and $\mathrm{Ti}$. The model atmosphere has $T_{\text {eff }}=8500 \mathrm{~K}, \log g=4$ and $X=0.72$ (Kurucz 1979).. We assume that convection is not suppressed by the magnetic field, but only slightly reduced relative to the Schwarzschild criterion (Babel 1992).

The results are shown in Fig. 4, for $\mathrm{Ca}, \mathrm{Sr}, \mathrm{Cr}, \mathrm{Mn}$ and $\mathrm{Ti}$ and for mass loss rates of 0 and $310^{-15} \mathrm{M}_{\odot} \mathrm{yr}^{-1}$. Except $\mathrm{Ti}$, all these elements have for solar abundances, $g_{\mathrm{rad}}<g$ in the upper most part of the atmosphere. Over the mass loss range considered, we note in Fig. 4 that the chemical abundances in the convection zone raise with the mass loss rate, but with strong variations from element to element. Inhomogeneous mass loss could thus produce abundance spots with varying contrast between the elements.

For mass loss rates larger than $\dot{M}=10^{-12} \mathrm{M}_{\odot} \mathrm{yr}^{-1}$, wind velocities are much larger than diffusion velocities both in the atmosphere and in the envelope as deep as $\Delta M / M=10^{-3}$. No chemical separation and thus no chemical anomalies can occur. 

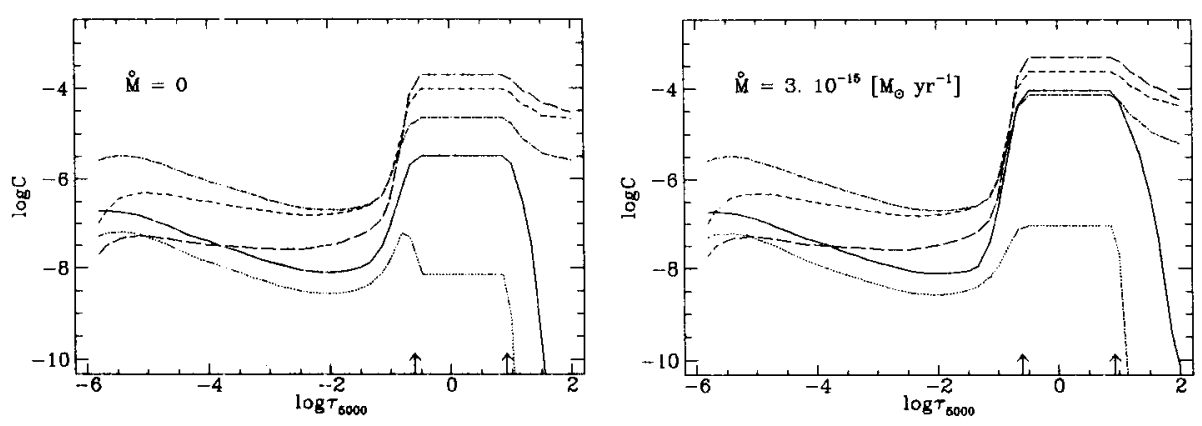

FIGURE IV Equilibrium abundance distributions in 53 Cam for a case without wind and for a mass loss rate of $\dot{M}=310^{-15} \mathrm{M}_{\odot} \mathrm{yr}^{-1}$ (Ca:solid line, Sr:dots, Mn:long dashes, Cr:short dashes, Ti:dot-long dashes). The arrows indicate the borders of the $\mathrm{H}$ II convection zone.

Comparison with observations

The most relevant way to compare the stratified abundance distributions of Fig. 4 with observations is to perform spectrum synthesis. We use the data from Landstreet (1988), kindly communicated by the author, for the visible domain. For the UV domain, we used IUE high-resolution spectra (Babel \& Lanz 1992). The magnetic and rotational parameters are from the previous analysis by Landstreet (1988). Abundance distributions are supposed to be axisymmetric relative to the magnetic axis. We calculated synthetic spectral line profiles in polarised radiative transfer for abundance distributions both inhomogeneous in surface and stratified vertically, with a modified version of the code zEEMAN (Landstreet 1988, Babel 1992).

Visible: Determination of the mass loss model from the Ca II $\mathrm{K}$ line

Calcium was used to determine the wind geometry of the diffusion-mass loss model for $53 \mathrm{Cam}$, for the following reasons:

- The calcium abundance distribution in the photosphere is very sensitive to the mass loss rate (see above) and slight variations of the mass loss rate or of the mass loss geometry could strongly modify both the intensity and the profiles of the $\mathrm{Ca}$ lines.

- The map of Ca derived by Landstreet (1988) from CaI is very contrasted on the stellar surface of $53 \mathrm{Cam}$.

- The CaII $\mathrm{K}$ line is very sensitive to abundance stratification. It must present. following our model, a very peculiar shape with broad wings and a very narrow core (Fig. 4 of Babel 1992). The peculiar profile of the $K$ line and its time variation permits also, as the magnetic poles successively pass near the line of sight, to separate the effects of stratification from the effects of abundance spots (see Bohlender \& Landstreet 1990).

From this analysis, we obtain that the time variation and the peculiar spectral profile of the $\mathrm{K}$ line are well reproduced by the presence of a mass loss rate of 
$\dot{M}=310^{-15} \mathrm{M}_{\odot} \mathrm{yr}^{-1}$ at the two poles and without mass loss in the equatorial region (Babel 1992). The wind geometry is shown in Fig. 5.

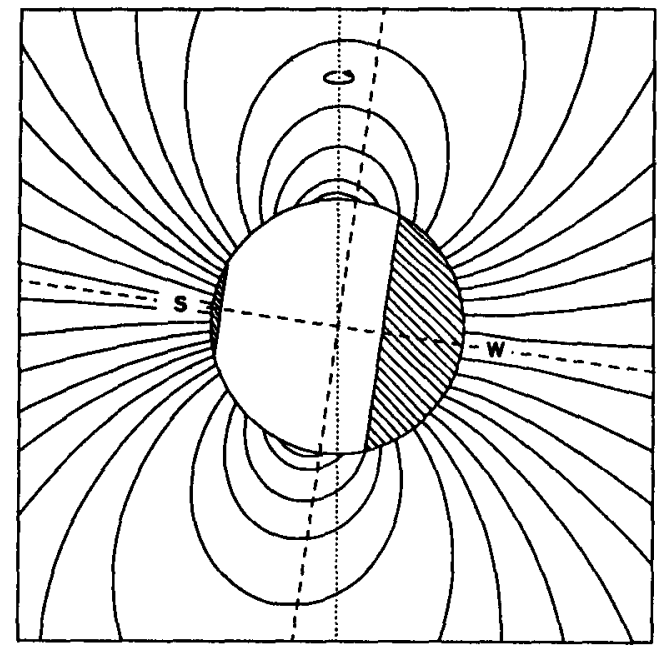

FIGURE V Mass outflow distribution on the surface of 53 Cam deduced from the profile and variation of the $\mathrm{Ca} I \mathrm{~K}$ line and from the diffusion-mass loss model ( $\dot{M}=310^{-15} \mathrm{M}_{\odot} \mathrm{yr}^{-1}$ : hatched areas; $\dot{M} \simeq 0.0$, elsewhere). The magnetic geometry is from Landstreet (1988). Labels $S$ and $W$ indicate respectively the strong pole $(-18000 \mathrm{G})$ and the weak pole $(4100 \mathrm{G})$. The small mass loss cap at the strong pole extend from $\alpha=0^{\circ}$ to $20^{\circ}$, while the cap around the weak pole goes from $\alpha=110^{\circ}$ to $180^{\circ}$. The size of the mass loss caps are quite well constrained by the large phase coverage. The rotation axis (with $i=64^{\circ}$ ) is represented by a dotted vertical line.

Others elements: With this model, the line profile of visible $\mathrm{Cr}$ lines could be very well reproduced. A good agreement is found also for Sr. Elements like Ti and $\mathrm{Mn}$ are not yet well accounted.

\section{UV abundances versus visible}

We perform subsequently a deep analysis of IUE high resolution spectra of 53 Cam (Babel \& Lanz 1992). From the domain $2500-3000 \AA$, abundances of $\mathrm{Cr}$, $\mathrm{Fe}, \mathrm{Mn}, \mathrm{Ti}$ and $\mathrm{Ca}$ were obtained for two phases near the alignment of the weak and strong poles. The most striking result of our UV analysis is the considerably lower abundances deduced from the $\mathrm{UV}$ domain for $\mathrm{Cr}$ and $\mathrm{Fe}$, than the values obtained by Landstreet (1988) from the visible spectrum. For chromium, we found, from different multiplets, abundances typically lower by 0.8 dex than in the visible. Similar results were obtained for iron, and again differences of about 0.7 to 0.9 dex with abundances deduced from the visible domain could be pointed out. We thus obtain a very good agreement between the predictions of the diffusion-mass loss model for chromium and both visible and UV $\mathrm{Cr}$ II line intensities (see Fig. 6 and Babel \& Lanz 1992). The very strong stratification predicted a priori by the model explains the ultraviolet lines well. This result rules out the presence of efficient turbulent processes in the line-forming region. 


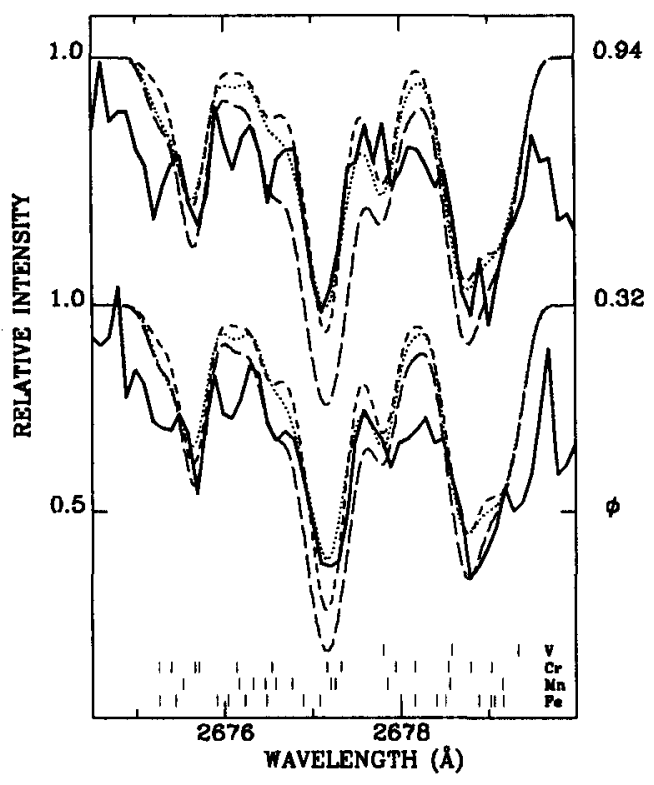

FIGURE VI IUE spectra (solid line) and spectrum syntheses for an uniform and non-stratified chromium abundance of $\log \epsilon_{\mathrm{Cr}_{r}}=-5$ (longdashed line), the best fit abundance $\log \epsilon_{\mathrm{Cr}}=-5.9$ (top), -5.5 (bottom, short dashes) and for the diffusion-mass loss model (dots). Phases are given on the right side.

\section{ABUNDANCES STRATIFICATION VERSUS $T_{\text {EFF }}$}

In stable atmospheres and for equilibrium abundance distributions, the abundance stratification is strongly linked with the variation of the wavelength distribution of the radiative flux in the atmosphere, as well as the variation of the absorption of the element. It also depends on saturation effects, non-local effects, etc... We compute equilibrium abundance distributions of $\mathrm{Ca}$ for stellar atmospheres with $T_{\text {eff }}$ between 7000 and $10000 \mathrm{~K}$. To illustrate the effect of radiative flux redistribution, we did not include homogeneization by convection nor ambipolar diffusion. In Fig. 7, we also made another approximation. As the radiative acceleration on $\mathrm{Ca}$ transmitted through the photoionisation of $\mathrm{Ca}$ II is poorly known and is important only at depths where convection occurs, we neglected here its contribution. In Fig. 7 are shown the $\mathrm{Ca}$ equilibrium abundance distributions as a function of $T_{\text {eff }}$ and $\log \tau_{5000}$. We obtain that the $\mathrm{Ca}$ abundance stratification, linked to the radiative acceleration transmitted to 


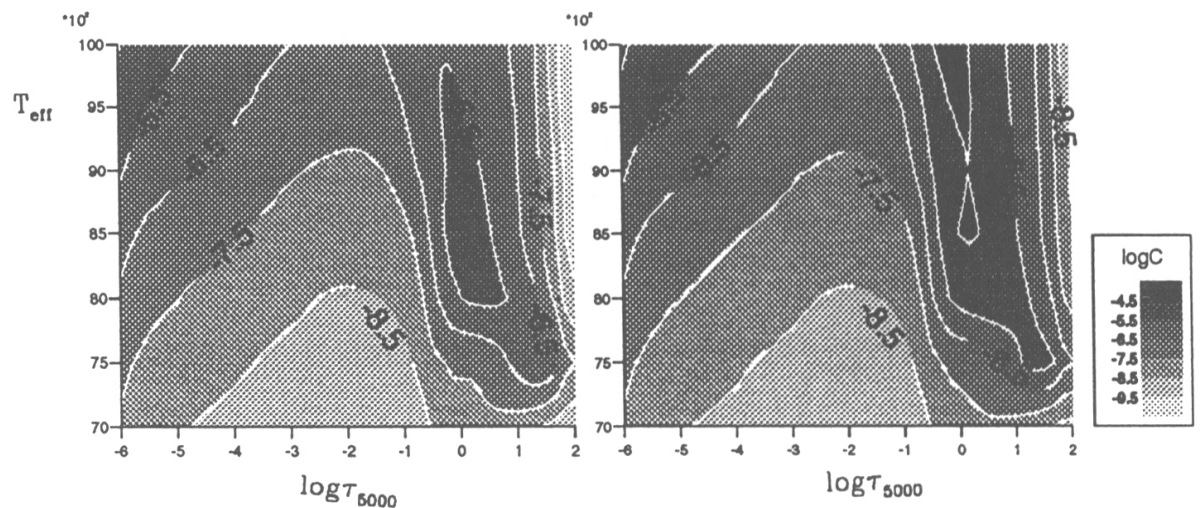

FIGURE VII Equilibrium abundance distribttions of $\mathrm{Ca}$ as a function of $T_{\text {eff }}$ and of the optical depth $\log \tau_{5000}$. The left panel is for $\dot{M}=0$, while the right panel is for a mass loss rate of $310^{-15} \mathrm{M}_{\odot} \mathrm{yr}^{-1}$.

spectral lines, is strongly dependent on the effective temperature. It is largest for effective temperature between 8000 and $9000 \mathrm{~K}$ being less important both in the cooler and hotter models. The right panel shows the effect of a small mass loss rate of $310^{-15} \mathrm{M}_{\odot} \mathrm{yr}^{-1}$. While the abundance below $\log \tau_{5000}=0.0$ (i.e. depth where $\mathrm{H}$ begin to be largely ionised) are then strongly enhanced, the $\mathrm{Ca}$ abundance in the upper layers is neither affected by proton drag nor by nonlocal effect of abundance stratification. The latter property can be explained by Fig. 8. This figure shows first that the radiative acceleration comes, even for saturate lines, from the core of the line and that the wing contribution is marginal. The main contribution to the radiative acceleration thus comes from region which are optically thick even in the upper layers, so that the radiative acceleration in the upper layers $\left(\log \tau_{5000} \leqslant-2\right.$ in Fig. 8) does not depend on the local abundance in the lower atmospheric layers $\left(\log \tau_{5000}>-1\right.$ in Fig. 8). As for $\mathrm{Sr}$, the presence of $\mathrm{H}$ II convection could modify the stratification, without modifying the abundance in the upper atmospheric layers.

From these results, we can make the following observational predictions.

- The most peculiar profiles of the $\mathrm{Ca}$ II $\mathrm{K}$ line (i.e. broad wings and narrow core) must be present in Ap stars with $T_{\text {eff }}$ between 8000 and $9000 \mathrm{~K}$. For $T_{\text {eff larger than }} 10000 \mathrm{~K}$, the stratification is strongly reduced and the effect on the profile is more subtle (see Fig.7 of Borsenberger et al. 1981).

- While the wings of the $\mathrm{Ca}$ II $\mathrm{K}$ line strongly depend on macroscopic processes (like convection or mass loss), the central part of the intensity profile of the $\mathrm{Ca}$ II $\mathrm{K}$ line (i.e. $0.3 \AA$ around line center) should be a common feature of cold Ap stars (except for modifications of the profiles linked with vsini) varying slowly with effective temperature and field intensity. In effect, it does only depend on radiative acceleration and not on mass loss nor on a variation of the extent of convection. 

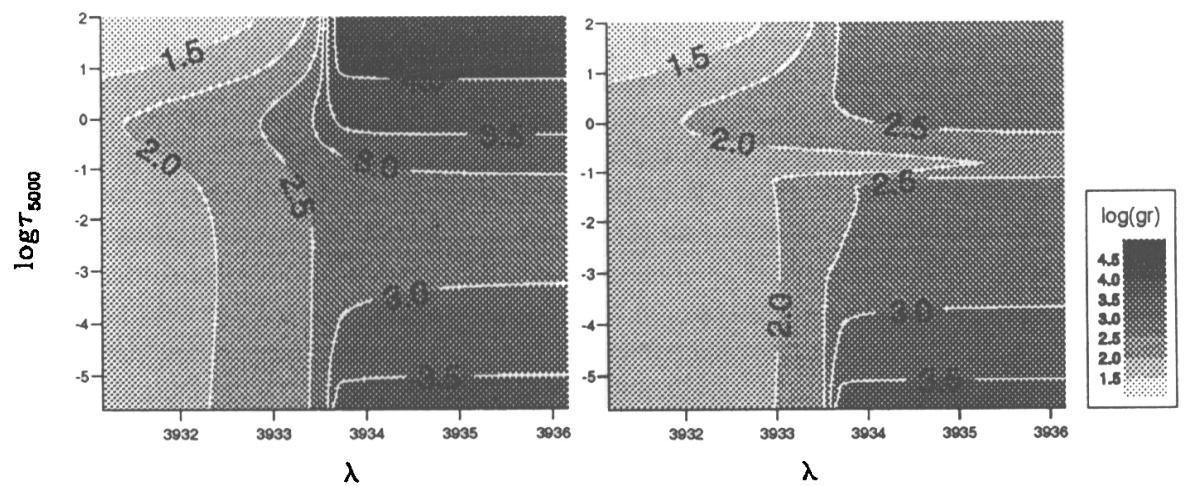

FIGURE VIII Wavelength cumulative contribution to the radiative acceleration of the $\mathrm{Ca}$ II $\mathrm{K}$ line as a function of the optical depth. Grey scale give the value of $\log \left(g_{\mathrm{rad}, \lambda}\right)$, where $g_{\mathrm{rad}, \lambda}$ is defined as $g_{\mathrm{rad}, \lambda}=\int_{0}^{\lambda} w_{\lambda^{\prime}} d \lambda^{\prime}$, with $w_{\lambda} d \lambda$ the monochromatic contribution to the radiative acceleration. The total radiative acceleration transmitted by the $\mathrm{Ca}$ II $\mathrm{K}$ line to $\mathrm{Ca}$ II could thus be read on the extreme right of each panel. The left panel is for a $\mathrm{Ca}$ abundance solar in the whole atmosphere. The right panel, is for an arbitrary stratified step function $\mathrm{Ca}$ abundance, with an overabundance of 2 dex below $\log \tau_{5000}=-1.0$ and a solar abundance above this depth.

\section{STRATIFICATION OF CALCIUM IN $\beta$ CRB AND HD 191742?}

Since more than 30 years, the peculiarity of the Ca II K line (3933.7 $\AA$ ) profile in Ap stars, was noted by several authors (e.g. Babcock 1958 and Wolff 1983). The results of the preceding sections lead us to investigate this problem in details, with high resolution and high signal-to-noise spectra. 21 "cold" Ap, with $T_{\text {eff }}$ generally lower than $10000 \mathrm{~K}$, were measured at OHP in april 1992 with Aurelie. The very peculiar Ca II $\mathrm{K}$ line profile observed on two objects, $\beta \mathrm{CrB}$ and $\mathrm{HD}$ 191742 (already noted as peculiar by Babcock 1958), are shown in Fig. 9. The temperature of these two stars is near $8000 \mathrm{~K}$. For $\beta \mathrm{CrB}$, most spectroscopic, photometric and bolometric determinations give a temperature between 7500 and $8000 \mathrm{~K}$. We obtain here a good fit of $\mathrm{H} \epsilon$ for $T_{\text {eff }}=8000 \mathrm{~K}, \log g=4$, $\chi=0.72$. For HD 191742, we adopt the same parameters. The most important features of these two spectra are the following.

- The $\mathrm{K}$ line is very important and very peculiar "...with extremely sharp core and very shallow wings..." (from the description by Babcock 1958).

- The Ca II H line (at $3968 \AA$ ) does not affect the spectral domain between $\mathrm{H}$ and $\mathrm{H} \epsilon$.

These two features give very stringent constraints on the abundance distribution which can fit both lines. In effect, while both surface inhomogeneities or abundance stratification permit to fit any peculiar $\mathrm{K}$ line (see Fig. 9), they give very different features around the $H$ line. The possible discrimination of these two models by use of $\mathrm{H}$ and $\mathrm{K}$ line profiles is linked to the very different 

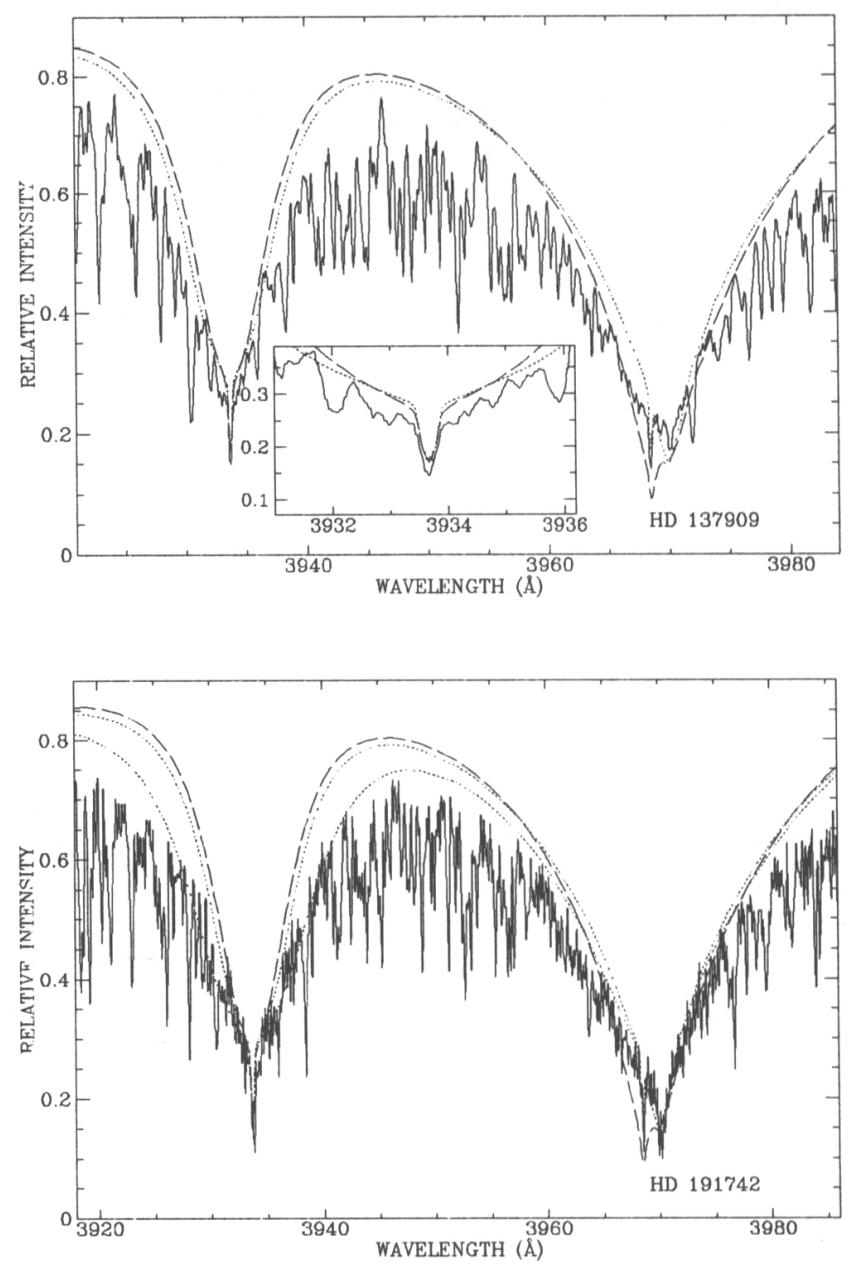

FIGURE IX Spectral domain around $\mathrm{K}$ and $\mathrm{H} \mathrm{Ca}$ In lines in $\beta \mathrm{CrB}$ and HD 191742. The dashed lines are for a model with spots of $\mathrm{Ca}$. The dotted lines are for spectral profiles obtained from the diffusion-mass loss model. The same models are used here for the two stars. For HD 191742, is also shown a spectral synthesis of the domain for a larger mass loss rate (and thus larger stratification) than in $\beta$ CrB. 
opacities $\kappa(\tau)$ between 3933 and $3968 \AA$, near the core of $H \epsilon$ (see a similar argument by Khoklova \& Topil'skaja, this meeting). To fit the wings of the $\mathrm{K}$ line, an inhomogeneous model without stratification requires spots with at least an overabundance of $\mathrm{Ca}$ by a factor of ten relative to the solar abundance and spots with an underabundance of $2.6 \mathrm{dex}$ ! The rich $\mathrm{Ca}$ spots necessarily create a strong distorsion of the profile of $\mathrm{H} \epsilon$ near $3968 \mathrm{~A}$. This is clearly not the case in these two stars. In contrast, the diffusion-mass loss model creates overabundances of $\mathrm{Ca}$ only below $\log \tau_{5000}=-1.0$ (see above) and thus at depths where the absorption from Balmer lines is maximum. The effect on $\mathrm{H} \epsilon$ of such a stratification is thus small. For both stars, we obtain that the diffusion-mass loss model permits to fit both the $\mathrm{K}$ and $\mathrm{H}$ lines. In Fig. 9, the convection is given by the Schwarzschild criterion and the mass loss rate are of $4.7-510^{-15} \mathrm{M}_{\odot} \mathrm{yr}^{-1}$.

\section{CONCLUSION}

The availability of high quality observations and the refinement of the diffusion models permits now to make severe comparisons and also, sometimes, to make predictions about line profiles and intensities. Several processes, which could be at the basis of abundance inhomogeneities have been discussed. It is now clear that diffusion processes alone cannot explain large surface inhomogeneities. In particular, diffusion can not explain the abundance maps on $53 \mathrm{Cam}$. In contrast, we have obtained that macroscopic processes affecting the major gas (hydrogen) are clearly very efficient in creating large inhomogeneities. Both mass loss, with its wind structure and/or mass loss rate, convection or ambipolar diffusion of hydrogen, can completely modify abundance patterns. Abundance maps give thus new opportunities to reveal the atmospheric structure of these peculiar stars, as they are signature of these processes. We have also shown, with observations at various $\kappa(\tau)$ that the vertical distribution of chemical elements could also been constrained. A detailed comparison, by spectrum synthesis, has been made between the diffusion-mass loss model and observations. The main results can be summarised as follows:

- An abundance stratification is clearly present in the photosphere of 53 Cam. The $\mathrm{K}$ line indicates a stratification of $\mathrm{Ca}$, while the $\mathrm{UV}$ and visible analysis give very strongs stratification of $\mathrm{Cr}$ and $\mathrm{Fe}$. The $\mathrm{Cr}$ and $\mathrm{Ca}$ stratification is well explained by the diffusion mass loss model. No model has yet been attempted for $\mathrm{Fe}$. For $\mathrm{Cr}$, the $\mathrm{UV}$ and visible line profiles are very well fitted with the strongly stratified abundance distribution from the diffusion-mass loss model. The central part of the peculiar $K$ line is also well fitted by our model. This part of the profile comes from region where the $\mathrm{Ca}$ abundance does not depend on any free parameter like mass loss or convection extent. These results support equilibrium abundance distributions (i.e. with $V_{t o t} \cong 0$ ) for $\mathrm{Ca}$ and $\mathrm{Cr}$ in $53 \mathrm{Cam}$.

- A calcium abundance stratification is undoubtly present in $\beta \mathrm{CrB}$ and HD 191742. Again, it can be explained by equilibrium distributions.

- The diffusion-mass loss model resolves nicely the longstanding problem of the peculiar $\mathrm{K}$ line profiles in Ap stars. In the context of this model, these peculiar profiles are interpreted as a signature of large abundance stratification of $\mathrm{Ca}$. 
It is clear, however, that large uncertainties remain. First, the model presented here is still rather simple. In particular, it is a first order model as the feedback of abundance distributions on the atmospheric structure is not included. Some more fundamental uncertainties are linked to the hydrodynamics. For example, the photospheric abundance of elements with $g_{\text {rad }}>g$ in the upper atmosphere, like $\mathrm{Ti}$ and the rare earths, can not yet be calculated satisfactorily as nothing is known about the wind structure and wind transition region. Is it a cold or hot wind? Is there some analogies with the solar wind and while some elements are trapped in the transition region others are dragged by the wind (Bürgi \& Geiss 1986) ? Detailed abundance analysis of rare earths could give very precious informations about the wind structure of magnetic stars. Another major difficulty comes still from our poor knowledge of the magnetic geometry, even if much progress has been done (Landstreet 1992). Abundance maps of several elements together with good magnetic geometry for several objects are clearly requested and could considerably help us to understand the physics of Ap stars.

\section{REFERENCES}

Abbott, D. C.:1979, in IAU Symp. 83, Mass loss and Evolution of O-type stars, ed. P. S. Conti and C. deLoore, (Dordrecht: Reidel), p. 237

Abbott, D. C.:1982, ApJ, 259, 282

Babcock H. W.:1958, ApJS, 3, 141

Babel, J.:1992, $A \& A, 258,449$

Babel, J., Lanz, T.:1992, $A \mathscr{E} A$, in press

Babel, J., Michaud, G.:1991a, ApJ, 366, 560 (BM91a)

Babel, J., Michaud, G.:1991b, $A \& A, 241,493$ (BM91b)

Babel, J., Michaud, G.:1991c, $A \& A, 248,155$ (BM91c)

Bohlender D. A., Landstreet J. D.:1990, ApJ, 358, L25

Borsenberger, J., Michaud, G., Praderie, F.:1981, ApJ, 243, 533

Bürgi, A., Geiss, J.:1986, Solar Phys., 103, 347

Burgers, J. M.:1969, Flow equations for Composite gases, Academic, New York

Fox, P. A., Theobald, M. L., Sofia, S.:1991, ApJ, 383, 860

Kurucz, R. L.:1979, ApJS, 40,1

Landstreet, J. D.:1988, ApJ, 326, 967

Landstreet, J. D.:1992, A\&A Rev., 4, 35

Linsky, J. L., Drake, S. A., Bastian, T. S.:1992, ApJ, 393, 341

Mégessier, C.:1984, $A \mathscr{G} A, 138,267$

Michaud, G., Babel, J.:1991, In Stellar atmospheres: beyond classical models, NATO Advance Workshop, Eds. Crivellari et al., Kluwer, p. 375

Michaud, G., Dupuis, J., Fontaine, G., Montmerle, T.:1987, ApJ, 322, 302

Michaud, G., Mégessier, C., Charland, Y.:1981, $A \& A, 103,244$

Michaud, G., Tarasick, D., Charland, Y., Pelletier, C.:1983, ApJ, 269, 239

Peterson, D. M., Theys J. C.:1981, ApJ, 244, 947

Shore, S. N., Brown, D. N., Sonneborn, G., Landstreet, J. D., Bohlender, D. A.:1990, ApJ, 348, 242

Unno, W.:1956, PASJ, 8, 108

Vauclair, S.:1975, $A \& A, 45,233$

Vauclair, S., Dolez, N., Gough, D. O.:1991, $A \& A, 252,618$

Vauclair, S., Hardrop, J., Peterson, D. M.:1979, ApJ, 227, 526

Wolff, S. C.:1983, The A-type Stars : Problems and Perspectives, NASA SP-463 